\section{REVISTA INTERNACIONAL DE CIENCIAS DEL DEPORTE International Journal of Sport Science}

Rev. int. cienc. deporte

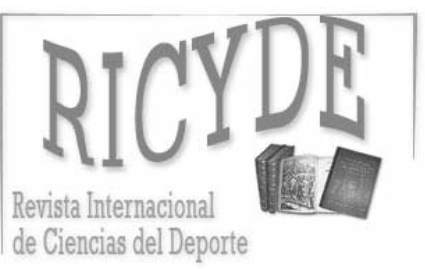

International Journal of Sport Science Volumen VI - Año VI

Páginas:141-153 ISSN:1885-3137 No 19 - Abril - 2010

\title{
Somatotipo y composición corporal en gimnastas de Trampolín masculino español de alto nivel. \\ Somatotype and body composition in elite male Spanish Trampoline.
}

\author{
Luís Arturo Gómez-Landero Rodríguez \\ Universidad Pablo de Olavide de Sevilla. España \\ Mercedes Vernetta Santana \\ Jesús López Bedoya \\ Universidad de Granada. España
}

\section{Resumen}

El presente estudio pretende describir y establecer datos de referencia de gimnastas españoles de la especialidad de Trampolín, centrándonos en el somatotipo y la composición corporal de varones en los distintos grupos de edad competitivos. Fueron evaluados 41 varones: 23 de categoría Sub-15 $(11,95 \pm 1,79$ años ) y 18 de categoría Absoluta $(20,72 \pm 4,66$ años), todos dentro de la élite nacional. Se estudiaron 15 variables antropométricas: peso, talla, 6 pliegues, 5 diámetros y 2 perímetros; todas obtenidas según el protocolo de la ISAK con investigadores certificados. El somatotipo fue calculado según el método de Heath-Carter y la composición corporal según las recomendaciones del GREC. En categoría Absoluta se obtuvo un $47,83 \%$ de masa muscular y $11,04 \%$ de grasa, con un somatotipo $2,4-4,7-2,8$; en Sub-15 se obtuvo un $46,37 \%, 10,38 \%$ de grasa y un somatotipo $2,8-5-2,8$. Los resultados presentan un biotipo medio mesomorfo balanceado con un SDI elevado de 3,54 en Sub-15 y 3,28 en Absoluta, distinto a los valores de referencia de gimnastas de la especialidad de Gimnasia Artística Masculina mucho más ecto-mesomorfos.

Palabras clave: trampolín; gimnasia; antropometría; somatotipo; composición corporal.

\section{Abstract}

The present study seeks to describe and establish reference data of Spanish Trampoline gymnasts, focusing on the somatotype and body composition of male gymnasts in several competitive age groups. 41 males: 23 Under-15 category (11.95 \pm 1.79 years) and 18 Absolute category (20.72 44.66 years), all in the national elite, were evaluated. 15 anthropometric variables were studied: body mass, height, 6 skinfolds, 5 breadths and 2 girths; all obtained according to the ISAK supervised by certified researches. The somatotype was calculated by the Heath-Carter method and body composition following GREC recommendations. In Absolute category the results showed $47.83 \%$ muscular mass and $11.04 \%$ of fat were obtained, with a somatotype $2.4-4.7-2.8$; while in Under-15 category, the figures were $46.37 \%, 10.37 \%$ and $2.8-5-2.8$. The results shows a balanced medium mesomorph somatotype with an elevated SDI of 3,54 in the Under-15 and 3,28 in Absolute, which are different from the data reference of male Gymnastics Artistic who are much more ecto-mesomorphic.

Key words: trampoline; gymnastics; anthropometry; somatotypes; body composition.

Correspondencia/correspondence: Luís Arturo Gómez-Landero Rodríguez

Universidad Pablo de Olavide de Sevilla. C/ Castañuelas no $35^{\circ}$ A. CP 41005 . Sevilla. España.

E-mail; arturogolaro@yahoo.es 
Gómez-Landero, L.A.; Vernetta, M.; López Bedoya, J. (2010). Somatotipo y composición corporal en el trampolín masculino español de alto nivel. Revista Internacional de Ciencias del Deporte. 19(6), 141-153. http://www.cafyd.com/REVISTA/01904.pdf

\section{Introducción}

on muy numerosos los estudios que han surgido ligados a la cineantropometría desde su aparición como ciencia, con un cuerpo doctrinal propio, en el Congreso Internacional de la Actividad Física de Montreal en 1976 (Chamorro, 1993). Como nos señala la Sociedad Internacional para el Avance de la Kinantropometría (ISAK, 2001), los estudios antropométricos incluyen una serie de herramientas con cómputos diversos para el análisis de datos, tales como el somatotipo, el fraccionamiento de la masa corporal, estimaciones de la proporcionalidad o la predicción de la densidad corporal con diversas ecuaciones de regresión; además presentan distintas orientaciones, que abarcan temáticas como el seguimiento y control de atletas, aspectos nutricionales, procesos de crecimiento y desarrollo o el rendimiento motriz.

Desde esta última perspectiva, Norton et al. (2004) señalan que dentro del amplio número de factores que influyen en el rendimiento deportivo, las medidas antropométricas en un deportista son variables que pueden jugar un rol importante en la determinación del éxito potencial en determinados deportes. Estos mismos autores, indican la necesidad de estudiar los perfiles morfológicos de los mejores deportistas de cada especialidad, como referencia sobre la que comparar.

De ahí que el objetivo de este estudio sea obtener un perfil morfológico de referencia, centrándose en el somatotipo y la composición corporal de gimnastas de categoría masculina del Trampolín de élite nacional, conocido popularmente como Cama Elástica e integrado como deporte gimnástico en la Federación Internacional de Gimnasia desde 1999 (Lokendahle, 1999). Con motivo de esta adhesión, esta especialidad deportiva ha sido recientemente incorporada al programa olímpico desde los Juegos Olímpicos (JJ.OO.) de Sydney 2000, incrementándose el número de publicaciones científicas en relación al mismo. Sin embargo, los trabajos antropométricos relacionados con este deporte han sido muy escasos, siendo ampliamente realizados en otras modalidades gimnásticas, como Gimnasia Artística (Masculina y Femenina) y Gimnasia Rítmica preferentemente.

Entre los más relevantes se encuentran los de Carter (1982 y 1984), que lideró un proyecto para analizar los perfiles antropométricos de los deportistas olímpicos en los Juegos Olímpicos de Montreal 1976, trabajo que incluye también datos referentes a los JJ.OO. de Tokio 1964, Méjico 1968 y Munich 1972. Un estudio más reciente publicado por Classens en 1991, estudia las características antropométricas de los gimnastas participantes en el Campeonato del Mundo de Rotterdam de 1987, incluyendo además datos de otros estudios relativos a Torneos Internacionales, Campeonatos Mundiales o Juegos Olímpicos realizados por diferentes autores.

El Grupo Español de Cineantropometría (GREC) ha estado utilizando entre otros, los valores de referencia aportados por Canda (1993) obtenidos en Centros de Medicina del Deporte Españoles. López Bedoya et al. (1993) hicieron una revisión de las publicaciones más relevantes relacionadas con las características morfológicas de las gimnastas de alto nivel. Rose et al. (1996) relacionaron el índice de masa corporal con el rendimiento en gimnastas de élite.

En otras especialidades gimnásticas se han encontrado trabajos realizados dentro del grupo de investigación CTS-171 de la Junta de Andalucía denominado "Análisis y Evaluación de la Actividad Física-Deportiva”, como el Aerobic Deportivo (López et al., 1999), el Tumbling y Trampolín (López et al., 2002), la Gimnasia Acrobática o Acrosport (Vernetta et al., 2003), la Gimnasia Rítmica (Fernández et al., 2006) y finalmente el Trampolín con los trabajos de Gómez-Landero et al (2004 y 2006). Todos ellos analizan las características morfológicas de 
Gómez-Landero, L.A.; Vernetta, M.; López Bedoya, J. (2010). Somatotipo y composición corporal en el trampolín masculino español de alto nivel. Revista Internacional de Ciencias del Deporte. 19(6), 141-153. http://www.cafyd.com/REVISTA/01904.pdf

los gimnastas de las distintas especialidades con un nivel de competencia mínimo en Campeonatos de España. Los artículos de Trampolín han supuesto un trabajo piloto previo al estudio que ahora se presenta, el cual se enmarca en un proyecto a nivel nacional denominado "Determinación del perfil motor, morfológico, funcional y psicológico en deportes gimnásticos para la construcción de baterías de test, aplicables a la detección y selección de talentos deportivos". El conocimiento de estos parámetros de rendimiento, permitirá obtener tablas antropométricas de referencia específicas para este deporte, que facilitarán tanto el proceso de detección y selección en esta modalidad gimnástica, como la realización de comparaciones entre diferentes trampolinistas en función de su categoría e, incluso, establecer diferencias con las otras modalidades gimnásticas.

Con todo lo anteriormente indicado, los objetivos de este estudio han sido:

o Obtener las medidas antropométricas de referencia en los trampolinistas de más alto nivel nacional.

o Analizar el somatotipo y la composición corporal de la muestra.

o Comparar los resultados obtenidos con los de otras especialidades gimnásticas.

\section{Material y Método}

\section{Participantes}

La población de estudio está compuesta por 41 trampolinistas varones. Todos presentan un nivel de competencia mínimo de Campeonato de España, siendo integrantes la gran mayoría de la Selección Nacional en las distintas categorías estudiadas y competidores a nivel internacional (Torneos Internacionales, Campeonatos de Europa y Campeonatos del Mundo). Estos gimnastas están entre los 45 mejores trampolinistas en el ranking nacional de 2006 (91,1\%) emitido por Real Federación Española de Gimnasia (RFEG). Todos aceptaron participar en el estudio de manera voluntaria, mediante consentimiento informado de acuerdo a las normas de ética para investigación en humanos, según los principios de la Declaración de Helsinki (World Medical Association, 2008).

Todos los sujetos se han agrupado según su categoría competitiva, en función de la Normativa de Trampolín 2006 de la RFEG propuesta en el Campeonato de España de Clubes, Campeonato de España de Selecciones Autonómicas y Copa de España, diferenciándose dos categorías:

\section{o Sub-15 (menos de 15 años) \\ o Absoluta (15 años o más)}

Las características generales de los grupos estudiados aparecen a continuación en la tabla 1.

Tabla 1. Características generales (edad, peso, talla e IMC) de los grupos estudiados.

\begin{tabular}{|c|c|c|c|c|c|}
\hline Grupos & Estadístico & $\begin{array}{c}\text { Edad } \\
\text { (años) }\end{array}$ & $\begin{array}{c}\text { Peso } \\
(\mathrm{kg})\end{array}$ & $\begin{array}{l}\text { Talla } \\
(\mathrm{cm})\end{array}$ & $\begin{array}{c}\mathrm{IMC} \\
\left(\mathrm{kg} / \mathrm{m}^{2}\right)\end{array}$ \\
\hline \multirow{2}{*}{$\begin{array}{c}\text { G } 1 \\
\text { (Sub-15) } \\
n=23\end{array}$} & Media & 11,96 & 38,80 & 143,99 & 18,44 \\
\hline & Des. Típica & 1,80 & 9,58 & 11,48 & 2,07 \\
\hline \multirow{2}{*}{$\begin{array}{c}\text { G } 2 \\
\text { (Absoluta) } \\
n=18\end{array}$} & Media & $20,72^{\star \star \star}$ & $64,10^{\star \star \star}$ & $171,04^{\star \star *}$ & 21,89 *** \\
\hline & Des. Típica & 4,66 & 8,01 & 4,07 & 2,42 \\
\hline \multirow{2}{*}{$\begin{array}{c}\text { Total } \\
n=41\end{array}$} & Media & 15,80 & 49,91 & 155,87 & 19,96 \\
\hline & Des. Típica & 5,50 & 15,47 & 16,26 & 2,80 \\
\hline
\end{tabular}


Gómez-Landero, L.A.; Vernetta, M.; López Bedoya, J. (2010). Somatotipo y composición corporal en el trampolín masculino español de alto nivel. Revista Internacional de Ciencias del Deporte. 19(6), 141-153. http://www.cafyd.com/REVISTA/01904.pdf

Ambos grupos, G1 y G2 (categoría Sub-15 y Absoluta), muestran diferencias estadísticamente significativas $(\mathrm{p}<0,001)$, presentando el $\mathrm{G} 2$ valores nítidamente superiores en edad, peso talla e IMC.

\section{Procedimiento}

El conjunto total de variables analizadas para este trabajo han sido: edad (años enteros), peso corporal (kg), estatura $(\mathrm{cm})$, seis pliegues cutáneos $(\mathrm{mm})$, subescapular, tríceps, supraespinal, abdominal, muslo frontal y pantorrilla; cinco diámetros $(\mathrm{cm})$, biacromial, bi-iliocrestal, epicondilar del húmero y del fémur y biestiloideo y dos perímetros $(\mathrm{cm})$ pantorrilla máxima y brazo flexionado y contraído.

A partir de este conjunto de medidas, se procesaron los datos para el análisis de la composición corporal siguiendo las recomendaciones del Grupo Español de Cineantropometría (GREC, 1993), que propone la estrategia de De Rose y Guimaraes (1980) basada en el modelo clásico de Matiegka (1921) para el cálculo de la composición corporal. Este método sigue para el cálculo del peso graso la fórmula de Faulkner (1968), para el peso óseo la de Von Doblen modificada por Rocha (1974) y para el peso residual la de Wurch (1974). Para el somatotipo se utilizó el método antropométrico de Heath-Carter (Carter, 2002), analizándose tanto la somatocarta como los componentes endomórficos, mesomórficos y ectomórficos.

La toma de datos se realizó la semana posterior a la Copa de España de Trampolín en el 2006, competición en la que participan una selección de los mejores en el ranking nacional. El momento de forma de cara a la toma de datos fue óptimo dada la proximidad de la competición. Se midió en el Centro de Alto Rendimiento de San Cugat en Barcelona, en el CAR de Trampolín de Albacete, en el centro de entrenamiento de Getafe (Madrid) y en el de Pontevedra (Galicia). Todas las medidas fueron realizadas por antropometristas acreditados por la ISAK para asegurar una mayor validez y fiabilidad durante el proceso. Las medidas obtenidas fueron registradas en una base de datos Excel 2002. Se estableció una distribución de frecuencias para cada variable, además de computar el mínimo y máximo hallado, mediana, media aritmética y desviación típica en todas las medidas en escala ordinal.

Se han establecido comparaciones de variables entre las dos categorías estudiadas y con otras especialidades gimnásticas. Para comprobar el tipo de distribución de cada variable, se aplicó la prueba de Kolmogorov-Smirnov. Si se cumplían los requisitos de normalidad se utilizó estadística paramétrica, con la prueba $t$ de Student en el caso de varianzas iguales y con la prueba t para varianzas heterogéneas (test de Welch). Si no se cumplían los requisitos de normalidad se utilizó estadística no paramétrica con la $U$ de Mann-Whitney. Para todas las comparaciones se estableció un intervalo de confianza del 95\%.

En el estudio de los somatotipos, se ha calculado el Índice de Dispersión del Somatotipo (SDI) para comprobar la homogeneidad de cada grupo, y para comparar los somatotipos medios de los distintos grupos la Distancia Morfogénica, término propuesto por el GREC para referirse al Somatotype attitudinal distance (SAD).

\section{Material}

Todo el material e instrumental utilizado para la toma de datos en las distintas pruebas, así como para el análisis posterior, estuvo acorde con las indicaciones de la International Society for the Advancement of the Kinanthropometry (ISAK): Báscula - impedanciómetro digital Tanita con precisión de 100g; compás de ramas curvas con precisión de 0,5 mm; calibre para pequeños diámetros Holtain con precisión de $1 \mathrm{~mm}$; tallímetro de pared con precisión de 1 mm; plicómetro Holtain con precisión de 0,2 mm; cinta antropométrica Lufkin de $2 \mathrm{~m}$ de 
Gómez-Landero, L.A.; Vernetta, M.; López Bedoya, J. (2010). Somatotipo y composición corporal en el trampolín masculino español de alto nivel. Revista Internacional de Ciencias del Deporte. 19(6), 141-153. http://www.cafyd.com/REVISTA/01904.pdf

longitud y precisión de $1 \mathrm{~mm}$; rotulador dermográfico; cajón antropométrico; hojas de registro; hoja de cálculo Excel diseñada al efecto para el procesamiento de datos; programa de análisis estadístico SPSS 15.0.

\section{Resultados}

En la figura 1 aparecen las somatocartas de todos los gimnastas, agrupadas según sus categorías competitivas. En la esquina superior derecha de cada gráfica aparece el índice de dispersión del somatotipo (SDI), calculado para valorar la homogeneidad de los grupos estudiados. Cuanto menor es el valor de SDI, menores diferencias existen entre los individuos del grupo estudiado y el grupo es más homogéneo. Si el resultado de SDI es $\geq 2$, se considera que existen diferencias significativas $(\mathrm{p}<0,05)$.

En este caso todos los valores del SDI están por encima de 2, apreciándose elevado nivel de heterogeneidad en cada grupo, con un SDI de 3,54 en Sub-15 y 3,28 en Absoluta. Al observar la dispersión en la nube de puntos puede apreciarse una tendencia entre un biotipo meosomorfo balanceado y ecto-mesomorfo en ambas categorías.
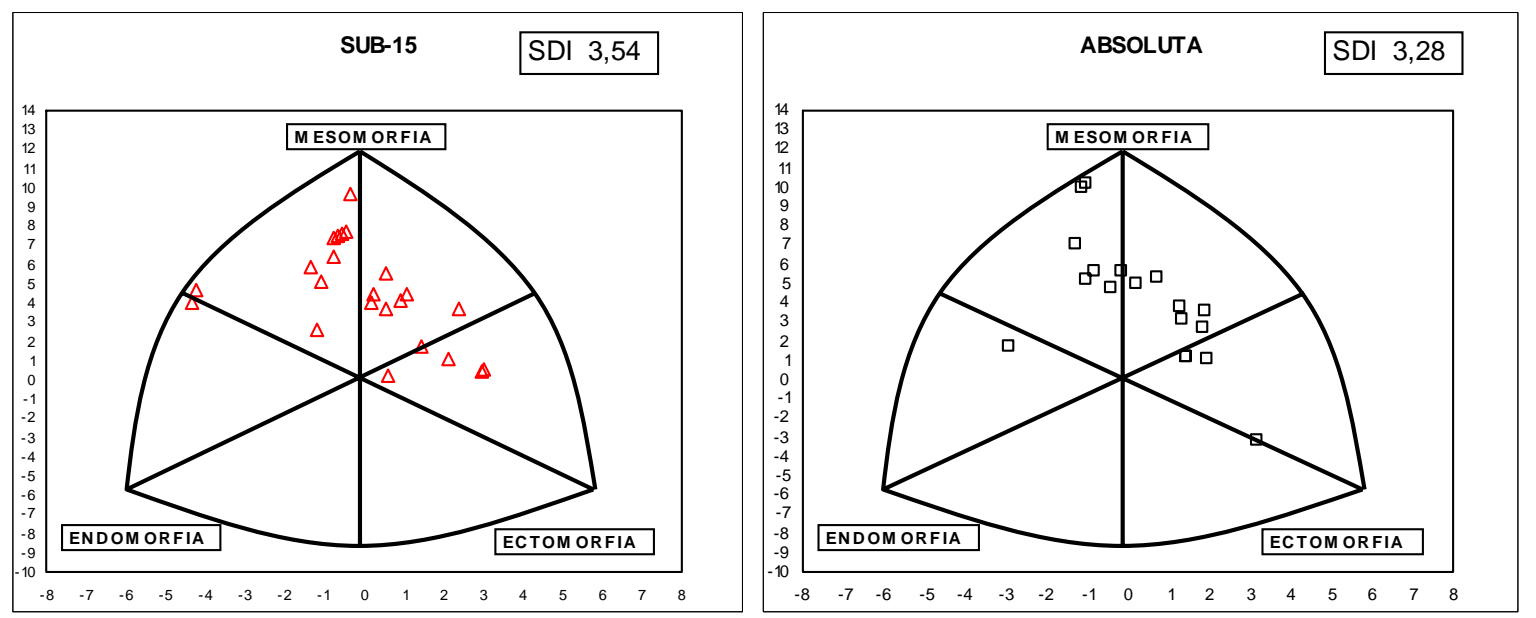

Figura 1. Somatocartas de todos los gimnastas estudiados agrupados por las categorías Sub-15 y Absoluta.

A continuación (tabla 2) aparecen las medias de ambos grupos y del total de la muestra $(\mathrm{n}=41)$ en los componentes del somatotipo (endomorfia, mesomorfia y ectomorfia) y en las coordenadas $(\mathrm{x}, \mathrm{y})$ de la somatocarta.

Tabla 2. Componentes del somatotipo y coordenadas de los grupos de trampolinistas estudiados.

\begin{tabular}{|c|l|c|c|c|c|c|}
\hline Grupos & Estadístico & $x$ & $y$ & Endomorfia & Mesomorfia & Ectomorfia \\
\hline $\begin{array}{c}\text { G 1 } \\
\text { (Sub-15) } \\
\mathrm{n}=23\end{array}$ & Media & 0,01 & 4,46 & 2,75 & 4,98 & 2,76 \\
\cline { 2 - 7 } & Des. Típica & 1,85 & 2,56 & 1,01 & 0,96 & 1,05 \\
\hline $\begin{array}{c}\text { G 2 } \\
\text { (Absoluta) } \\
\mathrm{n}=18\end{array}$ & Media & 0,36 & 4,11 & 2,44 & 4,69 & 2,81 \\
\cline { 2 - 7 } & Des. Típica & 1,54 & 3,24 & 0,63 & 1,17 & 1,12 \\
\hline $\begin{array}{c}\text { Total } \\
\mathrm{n}=41\end{array}$ & Media & 0,16 & 4,30 & 2,62 & 4,85 & 2,78 \\
\cline { 2 - 7 } & Des. Típica & 1,71 & 2,85 & 0,87 & 1,05 & 1,07 \\
\hline
\end{tabular}

\footnotetext{
* Diferencias estadísticamente significativas entre G1 y G2. Prueba t $(p<0,05)$
} 
Gómez-Landero, L.A.; Vernetta, M.; López Bedoya, J. (2010). Somatotipo y composición corporal en el trampolín masculino español de alto nivel. Revista Internacional de Ciencias del Deporte. 19(6), 141-153. http://www.cafyd.com/REVISTA/01904.pdf

En todos lo casos podemos apreciar cómo el componente mesomorfo prevalece respecto al endomorfo o ectomorfo. La media del somatotipo para toda la muestra masculina estudiada ( $\mathrm{n}=41)$ es 2,6 - 4,8 - 2,8 representando un biotipo mesomorfo-balanceado. Al comparar la categoría Sub-15 con la Absoluta apreciamos unos valores muy similares, siendo ligeramente más endomorfa y mesomorfa la Sub-15, y más ectomorfa la Absoluta, no encontrándose en ningún caso diferencias estadísticamente significativas entre ambos grupos.

Gráficamente podemos ver estas somatocartas en la figura 2.

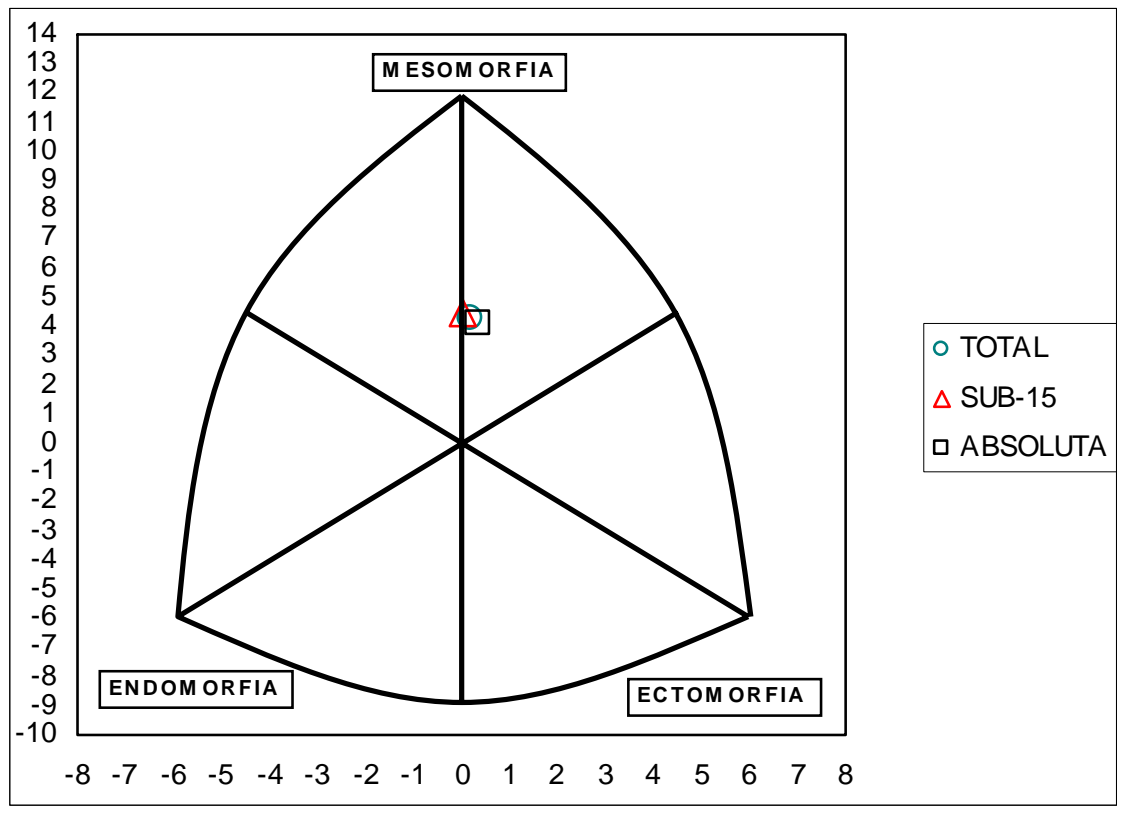

Figura 2. Somatocartas de los grupos estudiados y la media del total de la muestra.

En cuanto a la composición corporal podemos ver a continuación los resultados recogidos en la tabla 3 y representados en una gráfica de barras en la figura 3, diferenciando en cada caso el porcentaje del peso graso, óseo y muscular de los distintos grupos de edad

Tabla 3. Pesos y porcentajes de los cuatro compartimentos corporales estudiados (graso, muscular, óseo y residual) en los dos grupos.

\begin{tabular}{|c|c|c|c|c|c|c|c|c|c|}
\hline \multirow{2}{*}{ Grupos } & \multirow{2}{*}{ Estadístico } & \multicolumn{2}{|c|}{ MASA GRASA } & \multicolumn{2}{|c|}{ MASA MUSCULAR } & \multicolumn{2}{|c|}{ MASA ÓSEA } & \multicolumn{2}{|c|}{ MASA RESIDUAL } \\
\hline & & $\mathrm{Kg}$ & $\%$ & $\mathrm{Kg}$ & $\%$ & $\mathrm{Kg}$ & $\%$ & $\mathrm{Kg}$ & $\%$ \\
\hline \multirow{2}{*}{$\begin{array}{c}\text { G 1 } \\
(\text { Sub-15) } \\
n=23\end{array}$} & Media & 4,04 & 10,38 & 18,03 & 46,38 & 7,38 & 19,15 & 9,35 & 24,10 \\
\hline & Des. Típica & 1,16 & 1,48 & 4,65 & 1,42 & 1,69 & 1,50 & 2,31 & 0,00 \\
\hline \multirow{2}{*}{$\begin{array}{c}\text { G } 2 \\
\text { (Absoluta) } \\
\mathrm{n}=18 \\
\end{array}$} & Media & $7,13^{\star \star \star}$ & 11,04 & $30,69 * * *$ & $47,84^{\star \star}$ & $10,82^{\star \star *}$ & $17,02^{\star \star *}$ & $15,45^{\star \star \star}$ & 24,10 \\
\hline & Des. Típica & 1,57 & 1,36 & 4,24 & 1,43 & 0,84 & 1,50 & 1,93 & 0,00 \\
\hline \multirow{2}{*}{$\begin{array}{c}\text { Total } \\
\mathrm{n}=41\end{array}$} & Media & 5,40 & 10,67 & 23,59 & 47,02 & 8,89 & 18,21 & 12,03 & 24,10 \\
\hline & Des. Típica & 2,05 & 1,45 & 7,75 & 1,59 & 2,21 & 1,83 & 3,73 & 0,00 \\
\hline
\end{tabular}

** Diferencias estadísticamente significativas entre $G 1$ y G2. Prueba $t(p<0,005)$

*** Diferencias estadísticamente significativas entre G1 y G2. Prueba t $(p<0,001)$

El porcentaje de peso residual no aparece en la gráfica ya que se mantiene constante en los dos grupos al seguir el 24,1\% dado por Wurch (1974) para varones. 
Gómez-Landero, L.A.; Vernetta, M.; López Bedoya, J. (2010). Somatotipo y composición corporal en el trampolín masculino español de alto nivel. Revista Internacional de Ciencias del Deporte. 19(6), 141-153. http://www.cafyd.com/REVISTA/01904.pdf

Al comparar los porcentajes y pesos de los distintos compartimentos, se observan diferencias estadísticamente significativas entre ambos grupos. En el porcentaje graso se aprecia un valor más elevado en categoría Absoluta, sin embargo no existen diferencias significativas $(\mathrm{p}=0,147)$. En el porcentaje muscular sí encontramos sin embargo diferencias significativas $(\mathrm{p}<0,005)$. Con un $1,46 \%$ de masa muscular a favor de la categoría absoluta. Las diferencias son más notables con la masa ósea $(\mathrm{p}<0,001)$, que se presenta un $2,13 \%$ por encima en categoría Sub-15.

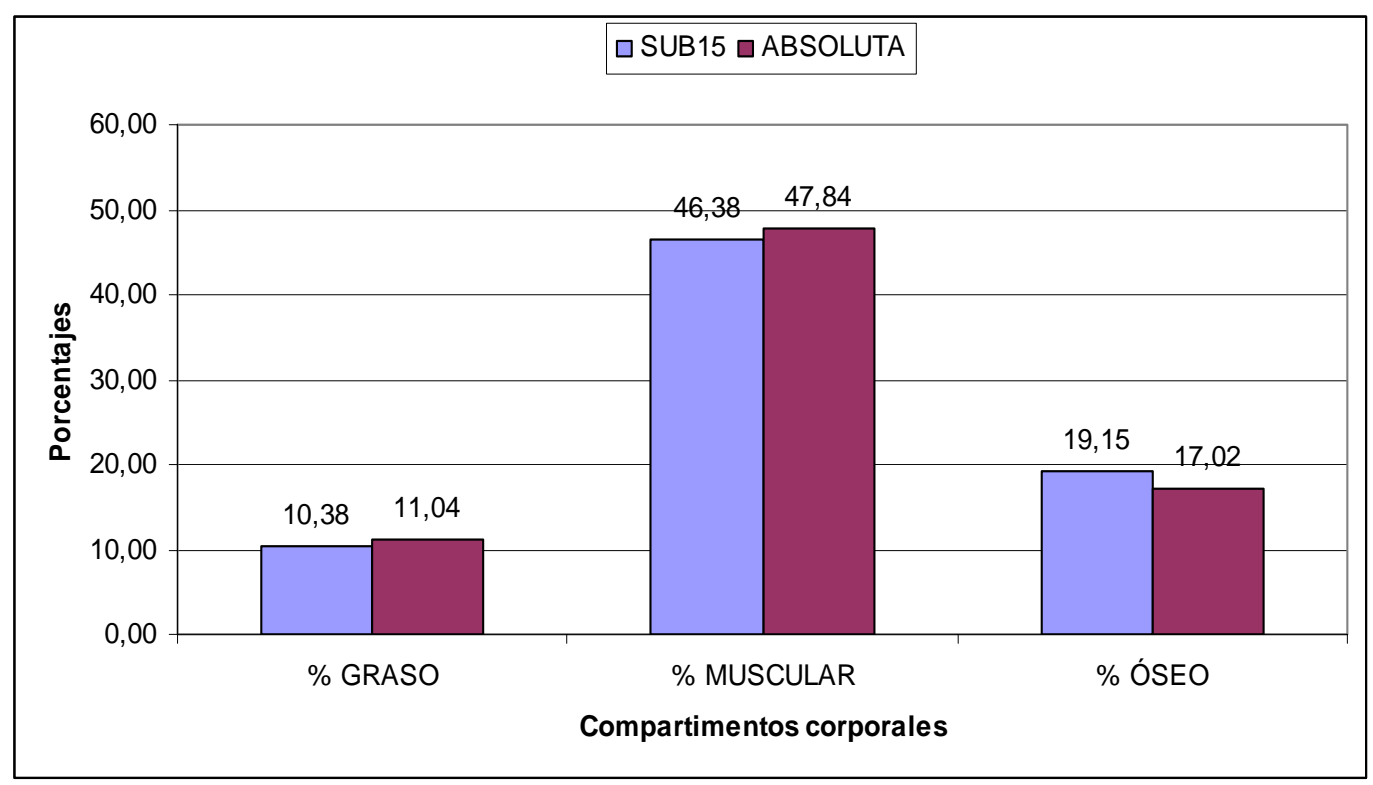

Figura 3. Composición corporal de los dos grupos de edades de los gimnastas estudiados

\section{Discusión}

Los resultados apuntan a que el somatotipo medio de los trampolinistas participantes en este trabajo muestra un predominio claro del componente mesomórfico, con valores similares en los componentes endomórficos y ectomórficos (2,6-4,9-2,8), conformando un somatotipo mesomorfo balanceado.

Se han apreciado diferencias notables entre ambas categorías competitivas en las medidas antropométricas generales. Las diferencias en cuanto a peso y talla entre la categoría Sub-15 y Absoluta han sido estadísticamente significativas para $\mathrm{p}<0,001$, justificándose la separación competitiva en ambos grupos de edad.

En menor medida, también se han apreciado diferencias al comparar la composición corporal de ambos grupos. Según los resultados, hay diferencias estadísticamente significativas en el porcentaje muscular (superior en Absoluta) y en el porcentaje óseo (superior en Sub-15). Estas diferencias pueden ser debidas en gran medida a los procesos madurativos característicos en la etapa evolutiva de la adolescencia (Rodríguez et al, 2004 y Moreno et al, 2006). Al comparar la categoría Sub-15 y la Absoluta se constatan grandes similitudes en sus somatotipos, con una distancia entre ambos muy baja (SDD=0,68) y sin diferencias significativas en ninguno de sus componentes, con una endormofia ligeramente superior en Sub-15 (2,8 frente a 2,4).

Resulta característico sin embargo, el alto grado de heterogeneidad dentro de cada categoría analizada (SDI elevado). 
Gómez-Landero, L.A.; Vernetta, M.; López Bedoya, J. (2010). Somatotipo y composición corporal en el trampolín masculino español de alto nivel. Revista Internacional de Ciencias del Deporte. 19(6), 141-153. http://www.cafyd.com/REVISTA/01904.pdf

Estos datos corroboran los estudios realizados por Gómez-Landero et al. (2004 y 2006) en categoría Absoluta, obteniéndose resultados muy similares.

Al comparar estos datos con los encontrados en la literatura científica relacionada, ha sido necesario recurrir a una modalidad gimnástica afín como la Gimnasia Artística Masculina (GAM), debido a la escasez de estudios antropométricos existentes de referencia sobre el Trampolín.

En la tabla 4 presentamos una comparativa entre la categoría Absoluta analizada, los datos de un estudio piloto con trampolinistas de la Selección Española (Gómez-Landero et al., 2004), y otros referidos a gimnastas de GAM (De Garay et al, 1974; Novak et al., 1977; Zaharieva et al., 1979; López et al., 1979; Carter, 1982; Norton, 1984; Claessens et al., 1991; Canda, 1993; Rodríguez y Berral, 2006).

Se han escogido los datos referentes a la categoría Absoluta al ser la que presenta una media de edad más cercana a las poblaciones de referencia encontradas. En la especialidad de Gimnasia Artística, la edad mínima para competir en categoría Absoluta a nivel internacional es de 16 años, siendo sin embargo de 18 años en el caso del Trampolín. Cabe señalar que el reducido número de la muestra de gimnastas españoles de $1993(\mathrm{n}=6)$ ha restado potencia a la discriminación estadística de las diferencias entre grupos. De la misma manera, no se han podido someter a comparación estadística las medias de las que no se disponían las correspondientes varianzas.

Exceptuando a la muestra de gimnastas españoles de 1993, el resto de poblaciones comparadas se sitúa en una media que ronda los 20 años desde los datos de 1984 hasta la actualidad. Los datos más antiguos presentan gimnastas con una edad media superior con diferencias estadísticamente significativas.

Tabla 4. Comparación de características antropométricas (valores medios y desviación típica) de gimnastas masculinos de élite, competidores a nivel internacional. CE= Campeonatos Europeos; JO= Juegos Olímpicos; CM= Campeonatos Mundiales: SN= Selección Nacional: CN= Campeonato Nacional.

\begin{tabular}{|c|c|c|c|c|c|c|c|c|c|c|c|c|c|}
\hline \multirow{2}{*}{\multicolumn{2}{|c|}{ VARIABLES }} & \multicolumn{10}{|c|}{ Gimnasia Artística Masculina } & \multicolumn{2}{|c|}{ Trampolín } \\
\hline & & \begin{tabular}{|l} 
Méjico JO \\
1968
\end{tabular} & \begin{tabular}{|l|} 
Munich JO \\
1972
\end{tabular} & \begin{tabular}{|l|} 
Varna CM \\
1974
\end{tabular} & $\begin{array}{l}\text { Montreal } \\
\text { JO } 1976\end{array}$ & $\begin{array}{l}\text { Santiago } \\
\mathrm{Cl} 1977\end{array}$ & \begin{tabular}{|l} 
China \\
1984
\end{tabular} & $\begin{array}{l}\text { Australia } \\
1984\end{array}$ & $\begin{array}{l}\text { Rotterdam } \\
\text { CM } 1987\end{array}$ & $\begin{array}{l}\text { C. Med. Dep. } \\
\text { Esp } 1993\end{array}$ & $\begin{array}{l}\text { Argentinos } \\
\text { SN } 2003\end{array}$ & $\begin{array}{l}\text { Españoles } \\
\text { SN } 2003\end{array}$ & $\begin{array}{l}\text { Españoles } \\
\text { CN } 2006\end{array}$ \\
\hline \multicolumn{2}{|c|}{ Muestra } & 28 & 126 & 126 & 11 & 33 & 19 & 7 & 165 & 6 & 25 & 7 & 18 \\
\hline \multirow{2}{*}{$\begin{array}{c}\text { Edad } \\
\text { (años) }\end{array}$} & Media & $23,6^{\star}$ & 24,7 & 23,8 & $25,4^{\star}$ & 26,1 & 19,8 & 19,8 & 21,9 & $16,3^{*}$ & 21,2 & 18,7 & 20,7 \\
\hline & D. T. & 3,8 & . & . & 4,6 & . & 3,3 & 2,7 & 2,4 & 1,2 & 3,3 & 3,7 & 4,7 \\
\hline \multirow{2}{*}{ Peso (kg) } & Media & 61,5 & 64,1 & 64,4 & 63,5 & 61,6 & $52,5^{\star}$ & 64,1 & 63,6 & 64,3 & 65,5 & 65,4 & 64,1 \\
\hline & D. T. & 5,5 & . & ${ }^{\circ}$ & 6,9 & . & 9,3 & 6,4 & 6,2 & 6,3 & 4,3 & 5,5 & 8,0 \\
\hline \multirow{2}{*}{ Talla $(\mathrm{cm})$} & Media & $167,4^{*}$ & 168,0 & 168,4 & 169,3 & 167,9 & $157,4^{\star}$ & 169,9 & $167^{*}$ & 167,1 & $166,2^{*}$ & 171,9 & 171,0 \\
\hline & D. T. & 5,7 & $\therefore$ & $\dot{.}$ & 5,6 & $\cdot$ & 8,7 & 5,5 & 6,3 & 4,8 & 4,7 & 3,3 & 4,1 \\
\hline \multirow{2}{*}{$\begin{array}{c}\text { Índice Masa } \\
\text { Corporal }\end{array}$} & Media & 23,4 & 22,7 & 22,7 & 22,2 & 21,9 & 21,2 & 22,2 & 22,8 & 23,0 & 23,7 & 22,1 & 21,9 \\
\hline & D. T. & $\cdot$ & . & . & . & . & $\cdot$ & . & . & . & . & 2,4 & 2,4 \\
\hline \multirow{2}{*}{$\begin{array}{c}\text { Sum_6 } 6 \\
\text { pliegues }(\mathrm{mm})\end{array}$} & Media & . & . & . & $32,9^{*}$ & . & . & 42,8 & . & 45,3 & $40,5^{*}$ &. & 55,5 \\
\hline & D. T. & . & . & . & 9,9 & 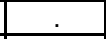 & 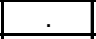 & . & . & 11,0 & 1,2 & 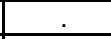 & 13,5 \\
\hline \multirow{2}{*}{ Endomorfia } & Media & $1,4^{*}$ & . & . & $1,4^{*}$ & 1,8 & $1,1^{*}$ & 1,8 & $1,5^{*}$ & 2,1 & 1,9 & 2,9 & 2,4 \\
\hline & D. T. & 0,3 & . & . & 0,5 & . & 0,3 & . & 0,3 & 0,5 & . & 0,4 & 0,6 \\
\hline \multirow{2}{*}{ Mesoforfia } & Media & $5,9^{*}$ & . & . & $5,8^{*}$ & 5,9 & $6,3^{*}$ & 6,2 & $5,6^{*}$ & 5,6 & 6,7 & 4,7 & 4,7 \\
\hline & D. T. & 0,8 & . &. & 0,8 &. & 1,1 & . & 0,7 & 1,0 &. & 1,5 & 1,2 \\
\hline \multirow{2}{*}{ Ectomorfia } & Media & 2,4 &. &. & 2,5 & 2,7 & 2,5 & 2,5 & $2,1^{*}$ & 2,0 & 1,6 & 2,7 & 2,8 \\
\hline & D. T. & 0,9 &. & . & 0,8 & . & 1,0 &. & 0,6 & 0,8 & . & 1,3 & 1,1 \\
\hline \multicolumn{2}{|c|}{$\begin{array}{l}\text { SAD respecto a la } \\
\text { muestra estudiada }\end{array}$} & 1,61 & . & & 1,52 & 1,35 & 2,08 & 1,64 & 1,45 & 1,24 & 2,38 & 0,51 & 0,00 \\
\hline
\end{tabular}

* Diferencias estadísticamente significativas, comparación con trampolinistas españoles CN 2006. Prueba t $(p<0,05)$

En las medidas generales referentes al peso y talla encontramos diferencias entre las referencias de GAM y el Trampolín masculino estudiado. La muestra estudiada presenta una media de altura de $171 \mathrm{~cm}$, muy similar a los trampolinistas de 2003 y superior a todos los 
Gómez-Landero, L.A.; Vernetta, M.; López Bedoya, J. (2010). Somatotipo y composición corporal en el trampolín masculino español de alto nivel. Revista Internacional de Ciencias del Deporte. 19(6), 141-153. http://www.cafyd.com/REVISTA/01904.pdf

datos de GAM, con diferencias significativas respecto a los gimnastas de Méjico 1968 y Rotterdam 1987, así como con lo gimnastas chinos y argentinos.

A pesar de esa diferencia en la talla, no aparecen sin embargo diferencias significativas en cuanto al peso, salvo con los gimnastas chinos; consecuentemente el Índice de Masa Corporal (IMC) parece inferior en los trampolinistas.

Éstos presentan unos valores superiores en todos los pliegues analizados respecto a los datos de referencia de GAM. Al comparar la suma de los 6 pliegues observamos diferencias notables: 55,49mm en los trampolinistas frente a los 32,9mm, 45,3mm y 40,5mm que presentan los gimnastas de Artística (diferencias significativas con Montreal 1976 y argentinos 2006). Estos valores junto a los del IMC, hacen suponer un mayor porcentaje graso en los trampolinistas en comparación a la GAM.

El perfil motor de la GAM presenta una variedad notable de situaciones de apoyo y suspensión en los distintos aparatos de que se compone (suelo, salto, barra fija, paralelas, caballo con arcos, anillas). Cada uno de estos aparatos tiene unas exigencias concretas, siendo la fuerza relativa al peso del gimnasta una variable vital para el rendimiento deportivo. Unos valores elevados de fuerza relativa pasan necesariamente por una composición corporal con un bajo porcentaje graso y un elevado porcentaje muscular (Faria y Faria, 1989).

$\mathrm{Al}$ analizar el somatotipo apreciamos que los trampolinistas estudiados no siguen ese perfil morfológico. La endomorfia de los trampolinistas es superior a todos los datos de referencia de GAM, mostrando diferencias estadísticamente significativas con 4 de las 5 muestras comparadas (1'4, 1'4, 1'1 y 1'5 frente a 2'4). Este dato se relaciona directamente con los mayores pliegues cutáneos obtenidos y el porcentaje de grasa, sugiriendo una menor importancia en el Trampolín Masculino de la masa libre de grasa respecto a la GAM.

Con el componente mesomórfico sucede al contrario, ya que todas las muestras de gimnastas presentan valores superiores, con diferencias significativas en los mismos casos anteriores (5’9, 5’8, 6’3 y 5'6 frente a 4'7). Este dato sostiene lo comentado anteriormente: el componente muscular es menos importante en Trampolín respecto a la GAM.

En la ectomorfia, la media de los trampolinistas estudiados es superior a todos los datos de GAM, aunque no de forma tan notable como en los componentes anteriores $\left(2^{\prime} 4,2^{\prime} 5,2^{\prime} 7\right.$, 2'5, 2’5, 2'1, 2'0 y 1'6 frente a 2'7), apareciendo solo diferencias significativas con los gimnastas del CM de Rotterdam de 1987. Estos resultados apuntan a un biotipo levemente más longilíneo de los trampolinistas estudiados respecto a los gimnastas de referencia.

Desde el punto de vista motor, el Trampolín se caracteriza por la interacción del gimnasta con un medio de gran capacidad elástica en el que ejecutará una sucesión de diez saltos acrobáticos con la acción predominante del tren inferior (Moreira y Peixoto, 2004). En estas circunstancias y ante la inexistencia de habilidades en apoyo o suspensión, no parece tan determinante un componente graso tan bajo y un componente muscular tan alto como en la GAM. Esto estaría en concordancia con la Ley de Hooke, según la cual la deformación del Trampolín es proporcional a la acción ejercida por el peso del gimnasta y los límites de la elasticidad de la composición del material (Monticelli, 1984).

Por otra parte, siguiendo a Zúñiga y de León (2007), según la evidencia experimental en diversas especialidades deportivas, existe una relación inversa entre masa grasa y rendimiento físico en actividades físicas que requieren el desplazamiento del cuerpo tanto en dirección vertical (como en el salto), como horizontal (la carrera).

Este tipo de aspectos relejan las diferencias que encontramos entre gimnastas de GAM y Trampolín. Los gimnastas de GAM, según López et al. (1993), de baja o media estatura y de 
Gómez-Landero, L.A.; Vernetta, M.; López Bedoya, J. (2010). Somatotipo y composición corporal en el trampolín masculino español de alto nivel. Revista Internacional de Ciencias del Deporte. 19(6), 141-153. http://www.cafyd.com/REVISTA/01904.pdf

reducido peso, reflejan una mayor predisposición para los esfuerzos que caracterizan a los movimientos gimnásticos y que se basan fundamentalmente en una elevada coordinación neuromuscular, dominio corporal en situaciones inhabituales, una elevada velocidad segmentaria y un índice alto de fuerza relativa.

Los trampolinistas con requerimientos motores aéreos similares (Vernetta y López, 2005), se ven favorecidos por el aumento del tiempo de vuelo, producto del peso del gimnasta y la elasticidad de la malla del trampolín, lo cual puede explicar pesos ligeramente mayores en esta población.

Todas las somatocartas de GAM y la de nuestra muestra de trampolinistas aparecen en la figura 4.

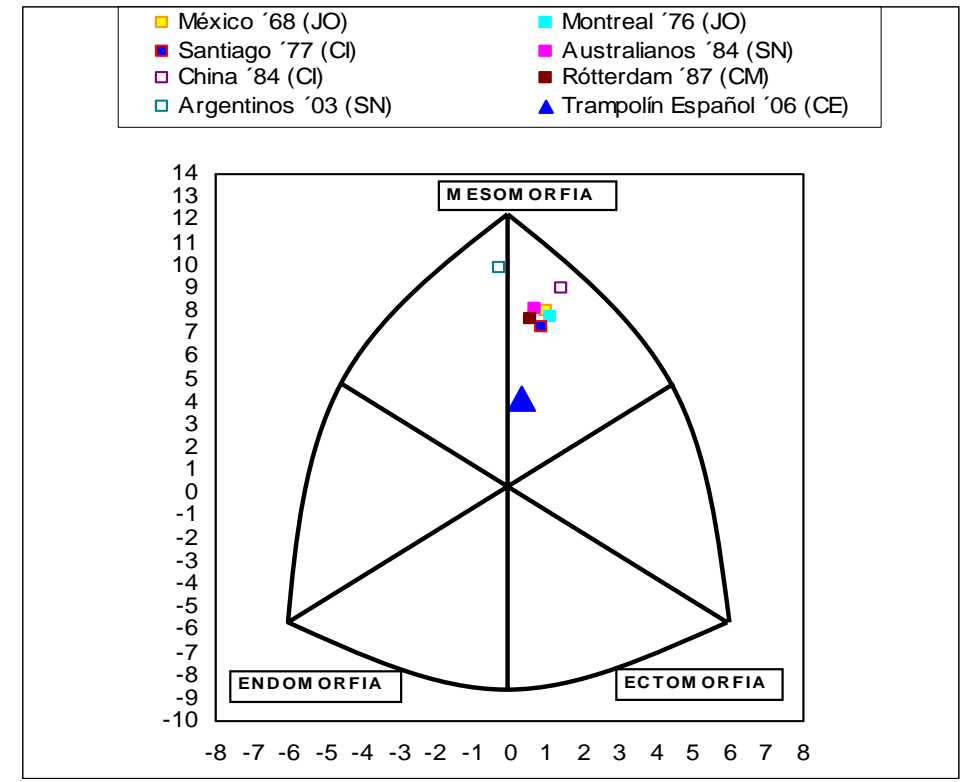

Figura 4. Somatocartas de diferentes muestras de gimnastas de Gimnasia Artística Masculina (GAM) y Trampolín (TR).

Todos los somatotipos de GAM se clasificarían como ecto-mesomorfos, menos el de los gimnastas argentinos, que presenta al igual que los trampolinistas un somatotipo mesomorfo balanceado. En la figura 4 podemos apreciar gráficamente como la mayor distancia SAD (tabla 4) aparece respecto a las muestras de chinos de 1984 y argentinos de 2003 (2,08 y 2,38 respectivamente); en el resto de muestras la distancia es baja, con valores por debajo de 2 . La muestra de trampolinistas españoles de 2003 es muy similar a la de 2006 con un SAD de 0,51.

\section{Conclusiones.}

o Las diferencias de peso y talla entre ambas categorías justifican desde el punto de vista antropométrico la separación competitiva de las mismas.

o La categoría Absoluta tiene mayor porcentaje muscular y la Sub-15 mayor porcentaje óseo.

o Las categorías competitivas analizadas en Trampolín masculino muestran un alto grado de heterogeneidad en sus somatotipos.

o La categoría Absoluta y Sub-15 presentan biotipos muy similares, con un somatotipo medio mesomorfo balanceado. 
o Los resultados obtenidos sugieren un perfil morfológico singular y característico en los gimnastas de Trampolín, con diferencias notables respecto a la Gimnasia Artística Masculina.

De cara a próximos estudios en esta línea de investigación se sugiere:

o Obtener datos antropométricos de referencia con poblaciones del más alto nivel internacional (finalistas en Campeonatos del Mundo o Juegos Olímpicos) y así poder establecer comparaciones.

o Estudiar la proporcionalidad corporal y analizar su posible influencia mecánica en la ejecución de habilidades propias del Trampolín.

o Realizar estudios correlacionales para discriminar la influencia de unos u otros parámetros morfológicos relacionándolos con índices de rendimiento, y así poder utilizarlos como un criterio más de cara a la detección y selección de talentos deportivos.

\section{Referencias bibliográfícas}

Canda, A. (1993) Valores cineantropométricos de referencia. En: Esparza Ross, F. editores. Monografías FEMEDE. Manual de Cineantropometría. 1ed. Madrid: Ed GREC FEMEDE, 171-213.

Carter, JEL. (1982) Physical Structure of Olympic Athletes. Part I. The Montreal Olympic Games Anthropological Project. Basel: Karger.

Carter, JEL.(1984) Physical Structure of Olympic Athletes. Part II. Kinanthropometry of Olympic Athletes. Basel: Karger.

Carter, JEL. (2002) The Heath-Carter Anthropometric Somatotype- Instruction Manual. Department of Exercise and Nutritional Sciences. San Diego State University. San Diego, CA. U.S.A. Surrey, Canada. March.

Claessens, L.A.; Veer, F.M.; Stijnen, V.; Lefevre, J.; Maes, H.; Steens, G.; Beunen, G. (1991) Anthropometric characteristics of outstanding male and female gymnasts. J Sports Sci; 9: 53-74.

López Bedoya, J.; Vernetta, M.; Cruz, J.C. (1999) Caracteristicas morfologicas y funcionales del Aerobic Deportivo. Apunts educacion fisica y deportes, n 55, pp 6065.

Chamorro M. (1993) Antecedentes históricos de la Cineantropometría. En: Esparza Ross, F. editores. Monografías FEMEDE. Manual de Cineantropometría. 1ed. Madrid: Ed GREC FEMEDE; 17-23.

De Garay, A.L.; Levine, L.; Carter, J.E.L. (1974) Genetic and Anthropological Studies of Olympic Athletes. New York: Academic Press.

De Rose, E. \& Guimaraes, A.C.A. (1980): Model for optimization of Somatotype in young athletes. En: Ostyn M., Beunen, G. \& Simons, J. Kinanthropometry II. Baltimore University Park Press.

Esparza Ross, F. (1993) Manual de Cineantropometría. Monografías FEMEDE. 1ed. Madrid: Ed GREC FEMEDE; pag 215.

Faria, I.E.; Faria, E.W. (1989) Relationship of the anthropometric and physical characteristics of male junior gymnasts to performance. J Sports Med Phys Fitness. Dec;29(4):369-78.

Faulkner, J.A. (1968) Physiology of swimming and diving. En: H. Falls (Ed), Exercise Phisiology, Baltimore: Academic Press. 
Gómez-Landero, L.A.; Vernetta, M.; López Bedoya, J. (2010). Somatotipo y composición corporal en el trampolín masculino español de alto nivel. Revista Internacional de Ciencias del Deporte. 19(6), 141-153. http://www.cafyd.com/REVISTA/01904.pdf

Fernández, E.; Vernetta, M.; López, J.; Gómez-Landero, L.A. (2006) Análisis de las características morfológicas en la selección andaluza de gimnasia rítmica deportiva. En González MA, Sánchez JA y Areces A (ed), IV Congreso Asoc. Esp. CC. de Deporte. A Coruńa. 24-27 oct.

Gómez-Landero LA, López J, Vernetta M, Fernández E. (2004) Análisis de las características morfológicas de la selección española masculina de trampolín. Lecturas: EF y Deportes. Revista digital, 74. Obtenido en http://www.efdeportes.com/efd74/tramp.htm (consultado el 29-1-2007).

Gómez-Landero, L.A., López, J, Vernetta, M., Fernández, E. (2006) Relaciones entre características funcionales y morfológicas en gimnastas de Trampolín. En González MA, Sánchez JA y Areces A (ed), IV Congreso Asoc. Esp. CC. de Deporte. A Coruńa. 24-27 oct.

ISAK. (2001) Estándares Internacionales para la Valoración Antropométrica. $1^{\text {a }}$ ed. Publ. ISAK.

Lokendahle, L. (1999) Historic Merger Paves the Way for Trampoline in the 2000 Olympics. ITIA Newsletter. Winter. Vol. 1, Issue 1.

Lopez, A.; Rojas. J.; García, E. (1979) Somatotype et composition ducorps chez les gymnastes de haut niveau. Cinesiologie, No. 72, 518.

López, J.; Gómez-Landero, L.A.; Jiménez, J.; Vernetta, M. (2002) Características morfológicas y funcionales en competidores de Tumbling y Trampolín. En León $\mathrm{K}$, Palomo A y Macías (ed.), Enseñanza y Entrenamiento de la Gimnasia y la Acrobacia, I Simposium Internacional de Actividades Gimnásticas y Acrobáticas (VII Simposium Nacional), Cáceres 11 y 12 abril.

López. J., Vernetta, M. \& Jiménez, J. (2003) Estudio cineantropométrico de gimnastas de Acrosport de nivel autonómico español. Lecturas: EF Y Deportes. Revista digita, 9. Obtenido en: http://www.efdeportes.com/efd67/acrosp.htm (consultado el 3-22007).

Lopez, J.; Vernetta, M.; De la Cruz, J.C. (1993) Características morfologicas y proceso de maduracion de las gimnastas de alto nivel. Morphological traits and maturation process in top level female athletes. Archivos de Medicina del Deporte 10(37), Jan/Mar, 49-55.

Matiegka, J. (1921) The testing of physical efficiency. Am. J. Phys. Anthrop., 4, 223-230.

Monticelli, M. (1984) II minitrampolino elastico in ginnastica artistica. Società Stampa Sportiva. Roma.

Moreira, M.; Peixoto, C. (2004) Análise das interacçoes de tres técnicas gímnicas em modalidades diferenciadas. Formaçao desportiva: Perspectivas de estudios nos contextos escolar e desportivo; 139-163

Moreno, L.A.; Mesana, M.I.; González-Gross, M.; Gil, C.M.; Fleta, J.; Wärnberg, J.; Ruiz, J.; Sarría, A.; Marcos, A.; Bueno, M. (AVENA Study Group) (2006) Anthropometric body fat composition reference values in Spanish adolescents. The AVENA Study. Eur J Clin Nutr.; 60, 191-196.

Norton, K.; Olds, T.; Olive, S., \& Craig N. (2004) Anthropometry and Sports Performance. In Norton K, Olds T, editors. Anthropometrica. 4 ed. Sydney: University of New South Wales Press. p. 287-364.

Novak, L.P.; Woodward, W.A.; Bestit, C.; Mellerowicz, H. (1977) Working capacity, body composition, and anthropometry of Olympic female athletes. Journal of Sports Medicine and Physical Fitness, 17, 275-83.

Real Federación Española de Gimnasia (2006) Normativa nacional de competición. Gimnasia en Trampolín. Madrid: RFEG. 
Gómez-Landero, L.A.; Vernetta, M.; López Bedoya, J. (2010). Somatotipo y composición corporal en el trampolín masculino español de alto nivel. Revista Internacional de Ciencias del Deporte. 19(6), 141-153. http://www.cafyd.com/REVISTA/01904.pdf

Rocha, M.S.L. (1975) Peso óseo do brasileiro de ambos os sexos de 17 a 25 anhos. Arquivos Anatomía e Antropología, 1, 445-451.

Rodríguez, E.; Berral, F. (2006) Morphological assessment in elite argentinean male gymnasts. Revista Brasileira De Cineantropometria \& Desempenho Humano (Florianopolis) 8(4), 16-24.

Rodríguez, G., Moreno, L.A.; Blay, M.G.; Blay, V.A.; Garagorri, J.M.; Sarria, A., y Bueno M. (2004) Body composition in adolescents: measurements and metabolic aspects. International J ournal of Obesity 28, S54-S58.

Vernetta, M.; López Bedoya, J. (2005). El contenido motor de los deportes de trampolín. Lecturas: EF y Deportes. Revista digital, 90. Obtenido en http://www.efdeportes.com/efd90/tramp.htm (consultado el 22-4-2007).

World Medical Assocation (2008) Declaración de Helsinki de la Asociación Médica Mundial. Principios éticos para las investigaciones médicas en seres humanos. Obtenido en http://www.wma.net/ es/30publications/10policies/b3/17c_es.pdf (consultado el 202-2006).

Wurch, A. (1974) La femme et le sport. Medicine Sportive Francaise. 4, 1.

Zaharieva, E. ; Georgiev, N. ; Techechmedgiev, R. (1979) Recherches anthropometriques su les gymnasts masculins et feminins des XVIIIe championnats du monde de Varna (Bulgarie) de 1974. Cinesiologie, 18, 19-24.

Zúñiga, U.; de León, L. (2007) Somatotipo en futbolistas semiprofesionales clasificados por su posición de juego. RICYDE. Revista Internacional de Ciencias del Deporte; 3 (9), 29-36. 